\title{
LA ESTRUCTURA INFORMATIVA EN ESPAÑOL HABLADO: APROXIMACIÓN PRELIMINAR
}

\author{
INFORMATION STRUCTURE IN SPOKEN SPANISH: A PRELIMINARY APPROACH
}

\section{Ferran Robles Sabater}

\section{Pau Bertomeu-Pi}

\section{LA ESTRUCTURA INFORMATIVA EN ESPAÑOL}

El dosier temático que aquí prologamos está dedicado al estudio de la estructura informativa en el español hablado. Desde perspectivas diversas, estos cinco trabajos abordan el análisis de fenómenos relacionados con los diferentes modos en que se segmentan y distribuyen en el enunciado los diferentes tipos de referentes y contenidos, tanto de naturaleza proposicional como instruccional, que forman el discurso. Es conveniente, pues, que delimitemos en qué consiste esta parte fundamental de la lengua y a qué líneas de investigación ha dado lugar en la discusión gramatical reciente.

La noción de estructura informativa que hoy maneja la lingüística hispánica tiene su origen en las propuestas de Halliday (1967) para un análisis de los enunciados basado no en las relaciones sintácticas que mantienen sus constituyentes, sino en la posición que estos ocupan dentro del discurso. A pesar de ello, no puede afirmarse que este autor fuera el primero en aludir a la estructura informativa de la oración como concepto, puesto que sus planteamientos partían y encontraban acomodo en algunas de las ideas expresadas en los años previos por los lingüistas de la Escuela de Praga. Sin llegar a emplear los mismos términos que el gramático británico, se refirieron en sus trabajos a algunos de los fenómenos descritos por él mediante nociones como la perspectiva funcional de la oración (Daneš, 1964), el dinamismo comunicativo y la organización del enunciado (Firbas, 1964, 1971, entre otros).

Halliday identificó tres «áreas de elección sintáctica» que influyen en la realización de las oraciones: la transitividad, el modo y el tema. Sobre el último, apuntó que «se vincula a la estructura informativa de la oración; al estatus de sus constituyentes no como miembros de procesos extralingüísticos, sino como los componentes de un mensaje; a la relación de lo que se dice con aquello que lo ha precedido en el discurso y su organización interna dentro de un acto de comunicación»' (Halliday, 1967: 199). Estos principios constituyeron la base desde la que autores posteriores como Chafe (1976, 1994) o Prince (1981) desarrollarían la noción de information packaging, esto es, "ciertos mecanismos con los

\footnotetext{
${ }^{1}$ La traducción de las citas que reproducimos en este trabajo es nuestra.
} 
que un emisor acomoda su discurso a estados mentales transitorios de la mente de su interlocutor", en palabras de Chafe (1976: 28). Una metáfora sirve a este sintactista y analista del discurso para describir la estructura informativa del enunciado como un embalaje o, mejor aún, como la envoltura que delimita, da unidad y recubre el contenido proposicional que es presentado dentro de este: «empleo el término embalaje [...] queriendo dar a entender que los fenómenos que aquí se abordan están relacionados, en primera instancia, con el modo en que el mensaje es enviado y, solo de forma secundaria con el mensaje en sí, del mismo modo que el envoltorio de la pasta de dientes puede repercutir en las ventas con una relativa independencia de la calidad de la pasta de dientes que contenga» (Chafe, 1976: 28).

En su fase más incipiente, el estudio de la estructura informativa se centró en concretar qué fenómenos se asocian a la voluntad de un emisor de disponer los contenidos que pretende transmitir en un determinado orden y de caracterizar los referentes nocionales que presenta como una información nueva o consabida, como el foco de un contraste, el tópico del enunciado, la expresión de la toma de posición del emisor respecto del contenido del enunciado, etc. Es aquí donde hay que enmarcar los primeros trabajos de los autores hispánicos sobre esta temática. En ellos, se deja de lado el análisis del papel que los referentes materiales o nocionales de estos constituyentes juegan en los eventos descritos por las oraciones para observar de qué modo la posición de tales constituyentes incide en la construcción del discurso y en su desarrollo. Aquí situamos los estudios de Ariza (1978), Contreras (1978), Rivero (1978), Fant (1984), Silva-Corvalán (1984) o Vallduví (1992), entre otros.

La investigación desarrollada en las décadas siguientes se ha centrado en el análisis de las principales funciones informativas - tema/rema (o soporte/aporte), foco/trasfondo (o presuposición), tópico/comentario - y sus formas de expresión en las diferentes lenguas. Estos estudios se han visto influidos por las tendencias dominantes en la lingüística europea y norteamericana, siendo especialmente relevantes las aportaciones que toman como base los principios del Generativismo (Chomsky, 1976) y de la Gramática de Construcciones (en especial, Lambrecht, 1994). Todos ellos coinciden en apuntar que la estructura informativa del enunciado representa la expresión formal del modo en que una proposición se estructura pragmáticamente dentro del discurso (Leino, 2013: 330). Así, Lambrecht (1994: 5) la definirá como «el componente de la gramática oracional en el que las proposiciones, como representaciones conceptuales de un estado de cosas, se asocian a estructuras léxico-gramaticales de acuerdo con los estados mentales de los interlocutores que emplean e interpretan tales estructuras como unidades de información en determinados contextos discursivos».

La lingüística española de las dos últimas décadas ha visto proliferar diferentes líneas de investigación y aplicaciones metodológicas relacionadas con la estructura informativa y sus formas de expresión. Sin ánimo de ser exhaustivos, queremos mencionar algunas de las más determinantes para la investigación actual, que en muchos casos tienen su punto de partida en los artículos de referencia de Zubizarreta y Moreno incluidos en la Gramática descriptiva de la lengua española de Bosque y Demonte (1999), así como en la obra Temas, remas, focos, tópicos y comentarios de Gutiérrez (1997).

Una línea que se inició hace ya cuatro décadas y sigue teniendo continuidad en la lingüística actual es el estudio del orden de palabras en el español hablado y escrito. A ella han dedicado trabajos Contreras (1978), Reyes (1985), Romero (1985), Ocampo (1990), Fernández (1993), Hidalgo (2000), Hernando (2005), Padilla (2005) o Cuartero (2007). Otra 
vertiente que también cuenta con una larga trayectoria es la que parte de los postulados del Generativismo chomskiano. En ella se inscriben las indagaciones de Elordieta (2007, 2012), Villalba (2010) o Hernanz (2011) sobre fenómenos como la dislocación, la focalización, las relaciones entre sintaxis y prosodia y el papel discursivo de los márgenes del enunciado. En relación con este último aspecto, también destacan los trabajos de Hidalgo (2003), López Serena (2012), Pons y Estellés (2014) o Borreguero (2014, 2015) sobre la posición inicial del enunciado como espacio privilegiado de determinadas funciones informativas.

En cuanto a la relación entre la estructura informativa y la organización del discurso, son especialmente relevantes las contribuciones de Fuentes $(2007,2014,2018)$ en torno a la sintaxis del enunciado y los complementos periféricos, con especial atención en los últimos tiempos a las estructuras parentéticas, así como el modelo de segmentación del discurso en la comunicación oral de Briz y el Grupo Val.Es.Co. (2003, 2014). Por lo que se refiere a la lengua escrita, destacan las propuestas Ferrari y Borreguero (2015) para la división del enunciado en unidades de primer y segundo plano informativo que tienden a ocupar zonas concretas dentro de este y aportar distintos tipos de contenidos referenciales y procedimentales.

En íntima relación con esta temática se hallan también los numerosos estudios dedicados a la incidencia de los marcadores discursivos en la estructuración informativa del enunciado y del texto. Destacaremos los de Cortés y Camacho (2005), Portolés (2010), De Cesare y Borreguero (2014), López Serena (2017), Salameh, Estellés y Pons (2018) y Hassler (2019), entre otros. Finalmente, merecen una mención aparte las pesquisas sobre estos marcadores realizadas desde una novedosa perspectiva pragmática experimental por Loureda y el grupo Partículas Discursivas y Cognición (Loureda et alii, 2015, Loureda, Nadal y Recio, 2016, 2018).

\section{SOBRE LOS ARTÍ́CULOS DE ESTE DOSIER}

Los artículos que conforman este bloque temático representan cinco diferentes aproximaciones metodológicas al estudio de fenómenos relacionados con la expresión de la estructura informativa en español. En el primero, Lidia Latorre García examina la distribución de la información en la conversación coloquial a partir de la noción de subacto, la unidad estructural mínima en que puede segmentarse el discurso y que, a su vez, es el constituyente inmediato del que se componen los actos, que son las unidades fundamentales de la comunicación. Mediante la observación de tres fragmentos de conversación, la autora muestra la existencia de patrones claros que se refieren, por una parte, al número de subactos de los que constan los actos analizados y, por otra parte, al tipo de subactos que predominan y su posición dentro de los actos en relación con los contenidos de naturaleza proposicional o instruccional que expresan.

En el segundo, Francisco Raga Gimeno se pregunta por la variabilidad de la concordancia en las oraciones hendidas del español y el catalán. Para ello parte de la consideración de que estas estructuras comparten una función básica de identificación, que se superpone a la puramente predicativa, por lo que no pueden considerarse como meras variantes informativas-perceptivas de las oraciones predicativas. Tal mecanismo cognitivo de identificación se manifiesta de diferente manera y con matices diversos en los dos niveles que el autor reconoce: el referencial y el textual-enunciativo. En cada uno de ellos, Raga Gimeno describe las circunstancias en que se pone de manifiesto o, al contrario, tiende a desaparecer la concordancia de género y número, persona y presencia de la preposición 
entre el elemento focalizado y su antecedente. Finalmente, el autor dedica un apartado a reflexionar sobre las oraciones hendidas con que invariable, que se presentan como el resultado de un proceso avanzado de gramaticalización, caracterizado por una pérdida de concordancia del verbo copulativo y un orden predeterminado de la estructura con poca movilidad de sus constituyentes.

En el tercero, Sílvia Planas-Morales y Xavier Villalba analizan las diferencias en la partición de foco y trasfondo en los enunciados interrogativos del español y el catalán. En su busca de patrones productivos para la señalización de esta división, descartan que mecanismos comunes en catalán como la dislocación del trasfondo a la derecha sean particularmente habituales en español. En las interrogativas, esta lengua no tiende a explicitar el antecedente como modo de marcar la partición foco-trasfondo, especialmente cuando tiene función de sujeto. Por lo que se refiere al hallazgo de correspondencias entre la entonación y la realización in situ del trasfondo, los autores demuestran la existencia de aspectos que alejan la realización prosódica de las interrogativas con dislocación a la derecha del catalán y sus correlativas con foco explícito en español del patrón típico de este tipo de enunciados cuando son pronunciados en contextos comunicativos neutros.

En el cuarto, Gloria Uclés Ramada reflexiona sobre las propiedades y funciones textuales del marcador discursivo ¿no?, una partícula con eminente valor interactivo que ha sido descrita tradicionalmente como un apéndice conversacional con valor intersubjetivo comprobativo y al que estudios recientes atribuyen funciones fática y expresivo-apelativa. Según la autora, la capacidad de estructurar el discurso de ¿no? tiene origen en su valor interpersonal y en su naturaleza apelativa, que le confieren la capacidad de delimitar fragmentos dentro el discurso y anclar al receptor en diversos puntos de las intervenciones del emisor, no solo con una intención meramente fática sino también para atraer su atención hacia lo dicho anteriormente. Mediante el análisis de fragmentos de tres diferentes géneros orales (conversación, entrevista y monólogo), Uclés Ramada identifica las cuatro principales funciones de ¿no? como estructurador, así como también los tipos de movimientos discursivos que es capaz de delimitar y vehicular cuando cumple cada una de estas funciones.

Finalmente, Araceli López Serena y Sara González Márquez analizan los usos discursivos de la expresión no sé cuando no forma parte de una estructura de predicación con complemento directo. Para ello realizan un tripe análisis de un corpus de conversaciones orales, en el que se combinan los principios de la segmentación informativa del enunciado, de las figuras de sintaxis y del modelo de continuum de inmediatez/distancia comunicativa aplicado al estudio de los marcadores discursivos. La comparación de los resultados obtenidos mediante estos tres procedimientos permite postular la existencia de correlaciones entre valores pragmáticos concretos y la capacidad de un marcador como no sé de aparecer en ciertos tipos de unidades discursivas y ocupar lugares precisos dentro de estas, así como concurrir con determinadas figuras de sintaxis.

\section{REFERENCIAS BIBLIOGRÁFICAS}

ARIZA, Manuel (1978): «Contribución al estudio del orden de palabras en español», Anuario de Estudios Filológicos, 1, 11-42.

BORREGUERO, Margarita (2014): «Left periphery in discourse», en Dufter, Andreas \& de Toledo, Álvaro O., eds., Left peripheries in Spanish, Amsterdam, John Benjamins, 345-382.
BorREGUERO, Margarita (2015): «El gato, que ha tirado un vaso. ¿Construcciones escindidas en el español coloquial?», Revista Internacional de Lingüística Iberoamericana, 26, 99-114.

Briz, Antonio y Grupo VAL.Es.Co. (2003): «Un sistema de unidades para el estudio del lenguaje coloquial», Oralia, 6, 7-61. 
GRUPO VAL.ES.Co (2014): «Las unidades del discurso oral. La propuesta Val.Es.Co. de segmentación de la conversación (coloquial)», Estudios de Lingüística del Español, 35, 13-73 [en línea]: <http://infoling.org/elies/35/elies35.1.pdf>.

CHAFE, Wallace L. (1976): «Givenness, contrastiveness, definiteness, subjects, topics, and point of view», en Li, Charles, ed., Subject and topic, Nueva York, Academic Press, 2555.

CHAFE, Wallace L. (1994): Discourse, consciousness, and time, Chicago, University of Chicago Press.

CHOMSKY, Noam (1976): "Conditions on rules of grammar», Linguistic Analysis, 2, 303-351.

CONTRERAS, Heles (1978): El orden de las palabras en español, Madrid, Cátedra.

CORTÉS, Luis y Matilde CAMACHO (2005): Unidades de segmentación y marcadores del discurso, Madrid, Arco Libros.

CUARTERO, Juan Manuel (2007): «Sobre la naturaleza y el alcance de los factores que influyen en el orden de palabras», Lingüística Española Actual, 29/1, 101-118.

DANEŠ, František (1964): «A three-level approach to syntax», Travaux linguistiques de Prague, 1, 225-240.

De Cesare, Anna-Maria y Margarita BorReguero (2014): «The contribution of the Basel model to the description of polyfunctional discourse markers", en Pons, Salvador, ed., Discourse segmentation in Romance languages, Amsterdam, John Benjamins, 55-94.

ElORDIETA, Gorka (2007): «Segmental phonology and syntactic structure", en Ramchand, Gillian y Charles Reiss, eds., The Oxford handbook of linguistic interfaces, Oxford, Oxford University Press, 125-177.

ElordietA, Gorka y Aritz IRURTZUn (2012): «Movimiento y prosodia», en Gallego, Ángel y José María Brucart, eds., El movimiento de constituyentes, Madrid, Visor, 213-234.

FANT, Lars (1984): Estructura informativa en español: estudio sintáctico y entonativo, Uppsala, Almqvist \& Wiksell International.

FERNÁNDEZ, Olga (1993): "Sobre el orden de palabras en español», Cuadernos de Filología Hispánica, 11, 113-152.

FIRBAS, Jan (1964): «On defining the theme in fuctional sentence analysis", Travaux linguistiques de Prague, 1, 267-280.

FIRBAS, Jan (1971): "On the concept of communicative dynamism in the theory of functional sentence perspective», Sborník Prací Filosofické Fakulty Brnĕské University, 19, 135144.

Fuentes, Catalina (2007): Sintaxis del enunciado: los complementos periféricos, Madrid, Arco Libros.
FuENTES, Catalina (2014): «Los límites del enunciado", Estudios de Lingüística del Español, 35, 143-167.

FuentES, Catalina (2018): Parentéticos, Madrid, Arco Libros.

GUTIÉRREZ, Salvador (1997): Temas, remas, focos, tópicos y comentarios, Madrid, Arco Libros.

HALLIDAY, M.A.K. (1967): «Notes on transitivity and theme in English", Journal of Linguistics, 3/4, 119-244.

HeRnANDO, Luis Alberto (2005): «El orden de palabras en español», Revista de Filología, 23, 161-178.

HeRnANZ, Maria Lluïsa (2010): "Assertive bien in Spanish and the left periphery", en Benincà, Paola \& Munaro, Nicola, eds., Mapping the left periphery, Oxford, Oxford University Press, 1962.

HIDALGO, Raquel (2000): "Orden de palabras y conversación", en Girón, José Luis et alii, eds., Estudios ofrecidos al profesor José Jesús de Bustos Tovar, 1, Madrid, Universidad Complutense, 125-134.

Hidalgo, Raquel (2003): La tematización en el español hablado, Madrid, Gredos.

LAMBRECHT, Knud (1994): Information structure and sentence form: topic, focus, and the mental representations of discourse referents, Cambridge, Cambridge University Press.

LEINO, Jaakko (2013): «Information structure», en Hoffmann, Thomas y Graeme Trousdale, eds., The Oxford handbook of construction gramar, Oxford, Oxford University Press, 329-344.

LÓPEZ SERENA, Araceli (2012): «En los márgenes de la estructura informativa: la posición inicial del enunciado como base», Lingüística Española Actual, 34/2, 303-337.

LÓPEZ SERENA, Araceli (2017): «Hacer (cosas con) palabras: la discursividad como universal genérico-esencial del lenguaje», Círculo de Lingüística Aplicada a la Comunicación, 69, 175-216, http://dx.doi.org/10.5209/CLAC.55319.

LOUREDA, Óscar et alii (2015): «Focus particles in information processing: an experimental study on pragmatic scales with Spanish incluso", Linguistik Online, 71/2, http://dx.doi.org/10.13092/lo.71.1781.

LOUREDA, Óscar, NADAL, Laura e Inés RECIO (2016): «Partículas discursivas y cognición: por tanto y la conexión argumentativa», Romanistisches Jahrbuch, 67/1, 240-254 <https://doi.org/10.1515/roja-2016-0016>.

LOUREDA, Óscar, NADAL, Laura e Inés RECIO (2018): «Die Konnektoren des Spanischen: eine experimentelle Annährung", Deutsche Sprache, 46/1, 82-96.

MoReno, Juan Carlos (1999): «Las funciones informativas: las perífrasis de relativo y otras construcciones perifrásticas», en Bosque, Ignacio y Violeta Demonte, eds., Gramática 
descriptiva de la lengua española, 3, Madrid, Espasa, 4245-4302.

OCAMPO, Francisco (1990): «The pragmatics of word order in constructions with a verb and a subject», Hispanic Linguistics, 4/1, 87-127.

Padilla, Xosé A. (2005): Pragmática del orden de palabras, Alicante, Universidad de Alicante.

PONS, Salvador y Maria ESTELLÉS (2014): «Absolute initial position", en Pons, Salvador, ed., Discourse segmentation in Romance languages, Amsterdam, John Benjamins, 121155.

PORTOLÉS, José (2010): «Los marcadores del discurso y la estructura informativa», en Loureda, Óscar y Esperanza Acín, eds., Los estudios sobre marcadores del discurso, hoy, Madrid, Arco Libros, 281-325.

PrINCE, Ellen F. (1981): "Toward a taxonomy of given/new information», en Cole, Peter, ed., Radical pragmatics, Nueva York, Academic Press, 223-255.

REYES, Graciela (1985): «Orden de palabras y valor informativo en español», en Fernández, Julio et alii, eds., Philologica hispaniensia in honorem Manuel Alvar, 2, Madrid, Gredos, 567-588.
RIVERO, María Luisa (1978): «Topicalization and whmovement in Spanish", Linguistic Inquiry, 11, 363-393.

ROMERO, María Victoria (1985): «Orden de los elementos oracionales en español», Rilce, 1, 91-111.

SALAMEH, Shima, Estellés, Maria y Salvador Pons (2018): «Beyond the notion of periphery: an account of polyfunctional discourse markers within the Val.Es.Co. model of discourse segmentation", en Beeching, Kate et alii, eds., Positioning the self and others, Amsterdam, John Benjamins, 105-125.

SILVA-CORVALÁN, Carmen (1984): «Topicalización y pragmática del español», Revista Española de Lingüística, 14/1, 1-19.

VALLDUVí, Enric (1992): The informational component, Nueva York, Garland.

VILLALBA, Xavier (2010): El orden de las palabras en español, Barcelona, Castalia.

ZUBIZARRETA, María Luisa (1999): «Las funciones informativas: tema y foco», en Bosque, Ignacio y Violeta Demonte, eds., Gramática descriptiva de la lengua española, 3, Madrid, Espasa, 4215-4244. 